\title{
Répartition du DAPA dans le contenu duodénal de vaches
}

\author{
K Tanan ${ }^{1}$, G Blanchart 2, C Poncet ${ }^{3}$, JC Robert *, \\ G Dumont ${ }^{1}$, E Desarménien ${ }^{1}$ \\ 1 Rhone Poulenc Animal Nutrition, 03600 Commentry; \\ 2 INRA-ENSAIA, 54500 Vandoeuvre-lès-Nancy; \\ 3 INRA, station de recherche sur la nutrition des herbivores, \\ 63122 Saint-Genès-Champanelle, France
}

La teneur en acide diaminopimélique (DAPA) des bactéries libres du rumen ou du duodénum est souvent utilisée comme référence lors de l'estimation du flux duodénal de biomasse microbienne. Afin de savoir si cette référence est représentative du DAPA total duodénal, nous avons fractionné le contenu duodénal en ses différentes phases et dosé le DAPA total dans chacune d'elle.

Trois vaches laitières taries fistulées du rumen et du duodénum reçoivent une ration d'ensilage d'herbe et de concentré $(50 / 50$, "H") en période 1 et d'ensilage de maïs et de concentré $(70 / 30$, ( $\mathrm{Mm}$ ) en période 2, à raison de $8 \mathrm{~kg}$ de $\mathrm{MS} / \mathrm{j}$ distribués en 8 repas égaux. Le contenu duodénal est prélevé à $6,10,14$ et $18 \mathrm{~h}$ sur un jour et à 8,12 , 16 et $20 \mathrm{~h}$ le lendemain. Le regroupement des prélèvements de chaque journée est filtré $(300 \mu \mathrm{m})$. Le résidu, après 2 lavages $(\mathrm{NaCl} 9 \mathrm{~g} /)$ ), représente les grosses particules (GP). Le filtrat et les solutions de lavage sont centrifugés $(1000 \mathrm{~g}$, $20 \mathrm{~min}, 4^{\circ} \mathrm{C}$ ), le culot obtenu représente les petites particules (PP). Le surnageant est centrifugé (23 $000 \mathrm{~g}, 30 \mathrm{~min}, 4^{\circ} \mathrm{C}$ ) pour isoler les bactéries libres (BL) qui constituent le culot. Le surnageant de la centrifugation $23000 \mathrm{~g}$ représente la phase soluble (PS). Les différentes fractions isolées sont poolées par vache sur les $2 \mathrm{j}$ de collecte puis lyophilisées avant la mesure des teneurs en matière organique (MO), et azote ( $\mathrm{N}$ ). Après une oxydation performique puis une hydrolyse acide $\mathrm{HCl} 6 \mathrm{~N} 24 \mathrm{~h}$ de l'échantillon, le DAPA est isolé par HPLC puis dosé en sortie de colonne par photométrie à 570 $\mathrm{nm}$ après réaction avec la ninhydrine.

Les PP ont des teneurs très élevées en DAPA et représentent une part importante du DAPA total, $68,9 \%(\mathrm{H})$ et $73,2 \%(\mathrm{M})$. Cela confirme la forte colonisation bactérienne de cette classe de particules déjà observée au niveau des conte- nus du rumen et du duodénum (Yang et al, 1989). Les GP sont beaucoup plus pauvres en DAPA et elles représentent $11 \%(H)$ et $6,3 \%(M)$ du DAPA total. Les BL ont des teneurs élevées en DAPA mais elles ne constituent qu'une faible part du DAPA total, 3,3\% (H) et 7,2\% (M). Le DAPA de la phase soluble représente une part importante du DAPA total $16,8 \%(H)$ et $13,3(\mathrm{M})$, cependant l'origine de ce DAPA n'est pas connue. II peut provenir du rumen sous forme libre ou/et avoir été libéré par hydrolyse des corps microbiens dans la caillette. L'essai met en évidence la faible part du DAPA associé aux bactéries libres et pose le problème de leur représentativité comme échantillon bactérien de référence.

Yang WZ, Poncet C, Escobar A (1989) AJAS 2 (3), 394-395

Tableau I. Concentration et répartition du DAPA dans les différentes fractions du contenu duodénal (moyenne, $n=3$ ).

\begin{tabular}{|c|c|c|c|c|}
\hline & \multicolumn{2}{|c|}{$D A P A \% M O$} & \multicolumn{2}{|c|}{$D A P A \% D A P A$ total } \\
\hline & $H$ & $M$ & $H$ & $M$ \\
\hline $\begin{array}{l}\text { GP } \\
\text { PP } \\
\text { BL } \\
\text { PS }\end{array}$ & $\begin{array}{l}0,057 \mathrm{c} \\
0,272 \mathrm{a} \\
0,183 \mathrm{bA} \\
0,148 \mathrm{~b}\end{array}$ & $\begin{array}{l}0,016 \mathrm{~d} \\
0,235 \mathrm{~b} \\
0,323 \mathrm{aB} \\
0,128 \mathrm{c}\end{array}$ & $\begin{array}{c}11,0 \mathrm{bc} \\
68,9 \mathrm{a} \\
3,3 \mathrm{c} \\
16,8 \mathrm{~d}\end{array}$ & $\begin{array}{c}6,3 \mathrm{~d} \\
73,2 \mathrm{a} \\
7,2 \mathrm{~cd} \\
13,3 \mathrm{bc}\end{array}$ \\
\hline ETR & \multicolumn{2}{|c|}{0,028} & \multicolumn{2}{|c|}{2,9} \\
\hline
\end{tabular}

Effets significatifs $(P<0,05)$ du régime $(A, B)$, et de la fraction isolée $(a, b)$.

\footnotetext{
* Correspondance et tirés à part
} 